ARTICLE

\title{
Mass spectrometry reveals the chemistry of formaldehyde cross-linking in structured proteins
}

Tamar Tayri-Wilk ${ }^{1,2,5}$, Moriya Slavin ${ }^{1,5}$, Joanna Zamel ${ }^{1,5}$, Ayelet Blass ${ }^{1}$, Shon Cohen ${ }^{1}$, Alex Motzik ${ }^{1}$, Xue Sun (1) 1, Deborah E. Shalev ${ }^{3,4}$, Oren Ram ${ }^{1} \&$ Nir Kalisman (1) ${ }^{1 \times}$

Whole-cell cross-linking coupled to mass spectrometry is one of the few tools that can probe protein-protein interactions in intact cells. A very attractive reagent for this purpose is formaldehyde, a small molecule which is known to rapidly penetrate into all cellular compartments and to preserve the protein structure. In light of these benefits, it is surprising that identification of formaldehyde cross-links by mass spectrometry has so far been unsuccessful. Here we report mass spectrometry data that reveal formaldehyde cross-links to be the dimerization product of two formaldehyde-induced amino acid modifications. By integrating the revised mechanism into a customized search algorithm, we identify hundreds of cross-links from in situ formaldehyde fixation of human cells. Interestingly, many of the crosslinks could not be mapped onto known atomic structures, and thus provide new structural insights. These findings enhance the use of formaldehyde cross-linking and mass spectrometry for structural studies.

\footnotetext{
${ }^{1}$ Institute of Life Sciences, The Hebrew University of Jerusalem, Jerusalem 9190401, Israel. ${ }^{2}$ Institute of Chemistry, The Hebrew University of Jerusalem, Jerusalem 9190401, Israel. ${ }^{3}$ Wolfson Centre for Applied Structural Biology, The Hebrew University of Jerusalem, Jerusalem 9190401, Israel. ${ }^{4}$ Department of Pharmaceutical Engineering, Azrieli College of Engineering, Jerusalem, Israel. ${ }^{5}$ These authors contributed equally: Tamar Tayri-Wilk, Moriya Slavin,

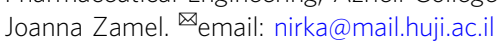


$\mathrm{F}$ ormaldehyde (FA) has been used as a fixative and preservative for many decades ${ }^{1,2}$. It is reactive toward both proteins and DNA, and forms inter-molecular cross-links between macromolecules ${ }^{3}$, as well as intra-molecular chemical modifications $s^{4,5}$. The high reactivity of FA together with its high permeability into cells and tissues has led to its use in numerous applications in biology, biotechnology, and medicine ${ }^{6}$. FA crosslinking of proteins is assumed to involve the formation of a methylene bridge between two proximal amino acids $\left(\mathrm{R}^{1}-\mathrm{CH}_{2}\right.$ $\left.\mathrm{R}^{2}\right)^{7,8}$. However, direct evidence to support this mechanism is sparse. In terms of mass, the methylene bridge adds $12 \mathrm{Da}$ (one carbon atom) to the total mass of the two cross-linked amino acids. Mass spectrometry has confirmed this $12 \mathrm{Da}$ addition to the masses of short linear peptides after FA incubation ${ }^{5,9-11}$. Yet, these studies were not able to identify pairs of peptides that were linked via methylene bridges. Thus, it is unclear whether the observed $12 \mathrm{Da}$ additions were bona fide cross-links or simply local modification of a single peptide.

Another puzzling fact is the lack of reports on the use of FA in the experimental technique of cross-linking coupled to mass spectrometry (XL-MS) ${ }^{12-14}$. In XL-MS, mass spectrometry identifies the protein residues that are linked based on the unique mass of the cross-linker. This information is then used to probe protein interactions ${ }^{15}$ and structures ${ }^{16}$. It seems fair to assume that if the methylene bridge reaction were easy to detect, FA would have been commonly used for in situ XL-MS ${ }^{17-20}$. Yet, we were only able to find reports of FA being used to stabilize protein complexes that were later cross-linked with a different reagent ${ }^{21,22}$. Given this lack of evidence, we hypothesize that FA cross-linking of proteins involves a different chemical mechanism. Identification of crosslinked peptides requires accurate knowledge of the chemical mechanism in order to calculate the mass of the cross-link product. Specifically, a search of mass spectrometry data with an incorrect mass of the adduct will not yield any identifications. Here we conduct an unbiased mass-spectrometric search for the FA adduct that leads to a different reaction product with a mass of $24 \mathrm{Da}$ and not the $12 \mathrm{Da}$ expected. This reaction only occurs in structured proteins (rather than peptides), perhaps explaining why earlier studies did not observe it.

\section{Results}

FA cross-linking of purified proteins. We first surveyed the FA cross-linking products that occur within structured proteins by cross-linking a mixture of three purified proteins (bovine serum albumin (BSA), Ovotransferrin, and a-Amylase). The mixture was incubated with FA for twenty minutes, and then quenched, denatured, digested by trypsin into peptides, and analyzed by mass spectrometry (Fig. 1). The general practice to identify a cross-link is by matching the measured mass to a theoretical total mass of the two peptides plus the mass of the cross-linker. Here, we did not limit our search to one predetermined cross-linker mass, but rather scanned through a range of possible masses. Figure 2 shows the number of cross-links that the scan identified for each cross-linker mass that was tested. It was surprising to see that the dominating reaction product adds exactly $24 \mathrm{Da}$ (two carbon atoms) to the total mass of the two peptides. This is different from the $12 \mathrm{Da}$ mass expected under the methylene bridge mechanism ${ }^{7}$. The broadening of the peak, which apparently includes reactions that add 25, 26 and 27 Daltons, is an artifact resulting from incorrect assignment of the mono-isotopic mass by the mass spectrometer (Supplementary Fig. 1). This artifact is common in XL-MS analysis ${ }^{23,24}$ and should not be interpreted as being due to alternative reaction products. We also tested a different brand of FA, which resulted in the same massscan profile (Supplementary Fig. 2a).

We find that the $24 \mathrm{Da}$ reaction is not two separate $12 \mathrm{Da}$ reactions occurring in parallel for two reasons: First, while one expects that a lower concentration of FA will show less of the 24 Da reaction and more of the $12 \mathrm{Da}$ reaction, we find that for both high and low concentrations of FA the mass-scan profiles are the same (Supplementary Fig. 2b). Second, ion species corresponding to mass additions of 36 or $48 \mathrm{Da}$ were not observed in Fig. 2, but such species should have occurred according to a parallel crosslinking model.

Further support for the uniqueness of the $24 \mathrm{Da}$ reaction is seen in the unusual fragmentation pattern of its MS/MS spectra (Fig. 3a-c). We find that the cross-link is highly susceptible to higher-energy collisional dissociation (HCD), and fragments in which it stayed intact could not be detected. Instead, it breaks symmetrically to give a mass addition of $12 \mathrm{Da}$ on each peptide. Peaks corresponding to the total mass of one of the peptides plus $12 \mathrm{Da}$ were among the most intense in the observed MS/MS spectra. The two peptides then break a second time to yield the standard b- and y-fragments as well as modified b- and yfragments with an additional $12 \mathrm{Da}$ mass. We find additional evidence for this two-step fragmentation model when we follow the change in fragmentation as a function of the normalized collision energy (Supplementary Fig. 4). Low collision energies are sufficient to break the cross-links, but are insufficient to break the stronger bonds of the $b$ - and $y$-fragments. The unique fragmentation pattern associated with the $24 \mathrm{Da}$ reaction resembles that of the cleavable cross-linkers frequently used in $\mathrm{XL}-\mathrm{MS}^{25}$. Yet, an important distinction is the $100 \%$ cleavage efficiency of the $24 \mathrm{Da}$ reaction, much higher than observed with other cleavable cross-linking reagents. The unusual fragmentation may partly explain why the $24 \mathrm{Da}$ reaction was not reported in previous FA studies.

With the understanding of the unique properties associated with XL-MS of FA, we designed an analysis application that is tailored specifically to identify the $24 \mathrm{Da}$ reaction and its subsequent MS/MS pattern. The application successfully identified cross-links in the three-protein mixture in a concentrationdependent manner (Fig. 3d). Interestingly, the application could also detect a small number of cross-links corresponding to the 12 $\mathrm{Da}$ reaction, but at a ratio of less than 1:7 relative to the $24 \mathrm{Da}$ reaction. Supplementary Data 1 lists an example of the identifications from one such cross-linking experiment. An attempt to analyze the same data with MeroX, an application tailored for cleavable cross-linkers ${ }^{26}$, gave only a third of the identifications (Supplementary Data 2), and these were a subset of our results. The smaller number is caused by certain features of FA cross-linking, such as multiple link sites, that are currently not supported by MeroX.

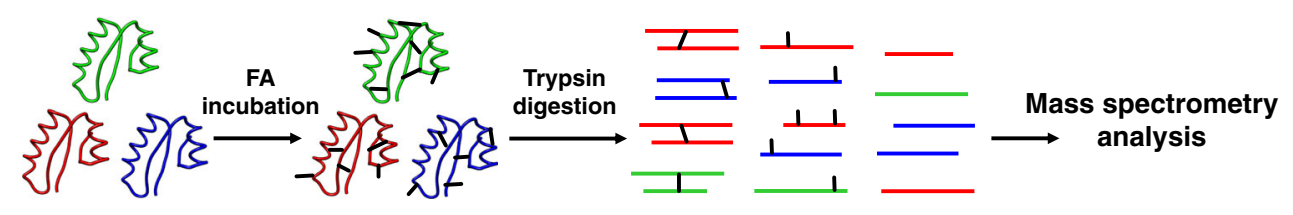

Fig. 1 The experimental setup for cross-linking of structured proteins by FA. The tryptic digest consists of a mixture of linear peptides, linear peptides with FA-induced modifications, and cross-linked peptide pairs. 


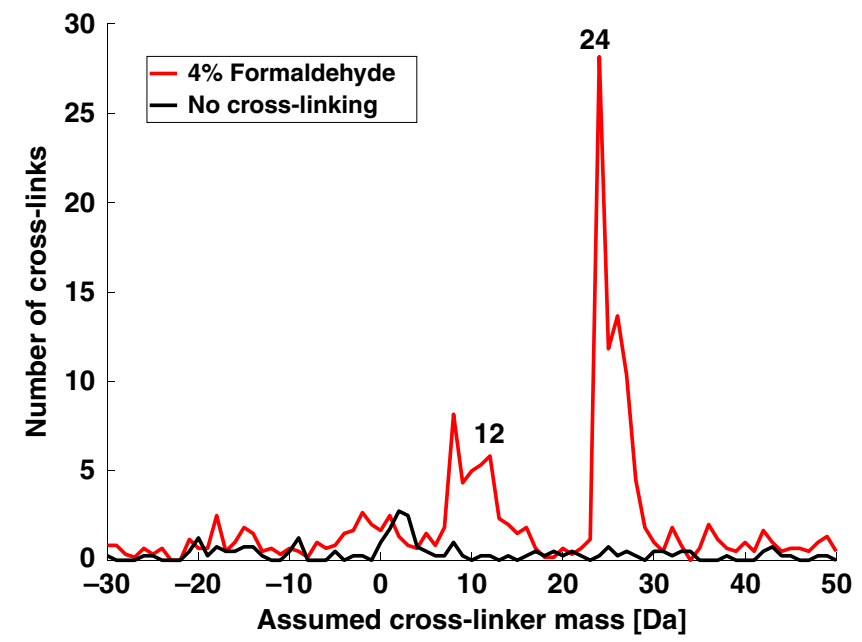

Fig. 2 FA cross-linking adds $24 \mathrm{Da}$ to the peptide pairs. Standard search for cross-links in mass spectrometry data from the setup that is described in Fig. 1. The $Y$-axis counts the number of identified cross-links. The search was repeated multiple times, each time assuming a different mass ( $X$-axis) being added by the cross-linking reaction to the total mass of the two linked peptides. Overlaid are mass scans from an experiment with high (4\%) FA concentration (red) and a control without cross-linking (black). Previously, FA cross-links were thought to be primarily methylene bridges that add 12 $\mathrm{Da}$, but in fact these contribute to very few identifications. Source data are provided as a Source Data file.
The modified +12 Da fragments in the MS/MS spectra allowed us to better characterize the amino acids that are most likely to partake in the reaction. To that end, we computationally modified in turn each residue along the cross-linked peptides, and determined which modification site was most compatible with the observed fragmentation pattern. The number of times each amino acid was found to be the most compatible was then normalized by dividing it by the total number of occurrences of that amino acid. This analysis clearly marks lysine and arginine residues to be the most prevalent in the $24 \mathrm{Da}$ reaction (Supplementary Fig. 5). The high reactivity of FA with these two amino acids is fully consistent with previous studies performed on peptides and single amino acids $5,7,10$. However, we note that a third of the identified cross-links involve at least one peptide that does not have a lysine residue. In these particular peptides aspartic acid and tyrosine residues are the most likely to be the linked residues. Interestingly, tyrosine was previously shown to be the third most reactive residue toward FA under certain conditions ${ }^{5}$. We conclude that the majority of FA crosslinks occur between lysine or arginine residues, but a significant fraction of cross-links also involve asparagine, histidine, aspartic acid, tyrosine, and glutamine residues.

The fragmentation pattern of the $24 \mathrm{Da}$ reaction does not enable identification of the two residues undergoing cross-linking. As a typical example, the fragmentation pattern of the peptide pair shown in Fig. 3b is consistent with the cross-link occurring on any of the first four residues in the upper (red) peptide. The localization is also ambiguous in the lower (blue) peptide as the a

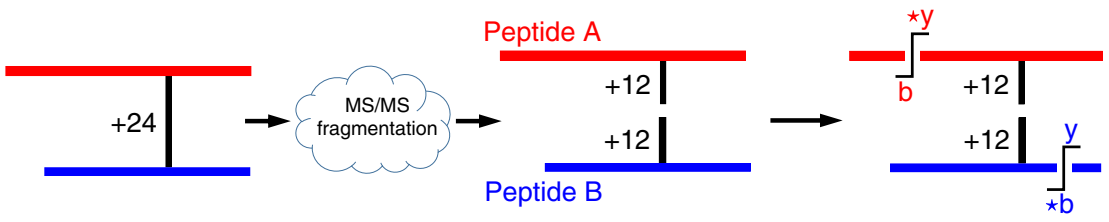

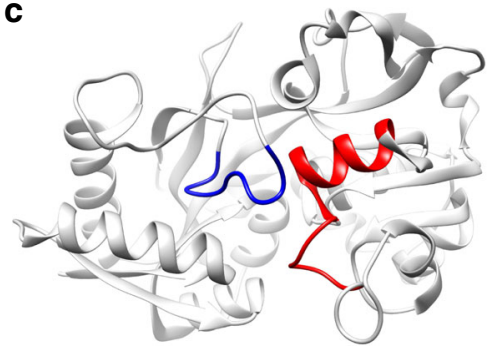
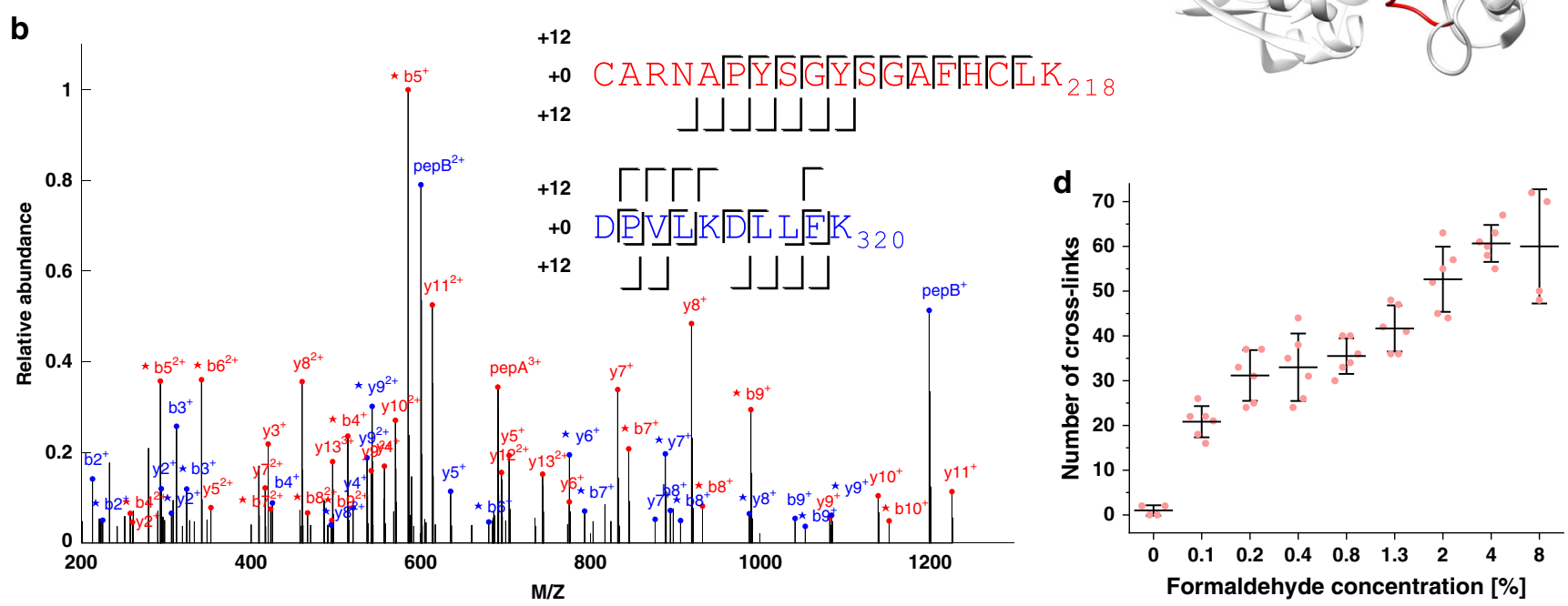

Fig. 3 Mass spectrometry characteristics of FA cross-links. a The MS/MS fragmentation of a formaldehyde cross-link breaks the ion to its two peptides, each with an additional mass of $12 \mathrm{Da}$. The two peptides then break a second time to produce $b$ - and $y$-fragments. $\mathbf{b}$ The MS/MS spectrum of a cross-link between two peptides of ovotransferrin. pepA and pepB are peaks matching the total mass of the corresponding peptides plus $12 \mathrm{Da}$. Peaks annotated with ${ }^{*} b$ or * $y$ match the masses of the corresponding b-and $y$-fragments plus $12 \mathrm{Da}$. For clarity, only peaks with intensities in the top quartile are shown. The annotation of the full spectrum is shown in Supplementary Fig. 3. c The two peptides identified in the above MS/MS spectrum are spatially close to each other in the crystallographic structure of ovotransferrin (PDBid 1OVT ${ }^{38}$ ). d The average number of cross-links identified for each formaldehyde concentration on a mixture of three proteins. Means and standard deviations calculated across three independent experiments, each with two technical replicates. The estimated false-detection rate is $3 \%$. Source data are provided as a Source Data file. 
first aspartic residue and the middle lysine-aspartic residues are all likely sites for the cross-link given the fragmentation. Therefore, the MS measurement shown in Fig. 3b may actually report a group of isomers of the same two peptides with different cross-link sites on each. This ambiguity usually does not occur with cross-linking reagents with high chemical specificity toward one particular amino acid type. The uncertainty in localizing the cross-link sites prevents the measurement of the exact distance spanned by a FA cross-link. Instead, we estimate the cross-link distance as the minimal $\mathrm{C} \alpha-\mathrm{C} \alpha$ distance between the two peptides on the protein structure. Supplementary Fig. 6 shows the histograms of the minimal distances observed for the crosslinks from several FA concentrations, and results from experiments with the cross-linking reagent disuccinimidyl suberate (DSS). This comparison indicates that FA cross-links are on average shorter than those of DSS.

FA modifications on linear peptides. As a control to the experiments on structured proteins, we incubated the peptide digest from the same three proteins with FA, and analyzed the products by mass spectrometry (Supplementary Fig. 7a). This analysis did not identify any cross-link between a pair of peptides in the digest. Yet, an analysis of single linear peptides found a high abundance of FA-related modifications (Supplementary Fig. $7 \mathrm{~b}, \mathrm{c}$ ). Contrary to the cross-links, which adds $24 \mathrm{Da}$, these modifications are dominated by a reaction that adds $12 \mathrm{Da}$ to the peptides. Just $20 \mathrm{~min}$ of incubation with $2 \% \mathrm{FA}$, is sufficient to form peptides with a single $12 \mathrm{Da}$ modification at significant numbers. These modifications were nearly absent when the digest was not treated with FA (No XL), and can therefore be attributed to the FA reactivity. Peptides with multiple modifications in parallel $(24,36,48$, and $60 \mathrm{Da})$ were also frequent, and increased in frequency at longer incubation times. Such modifications are fully consistent with observations of previous mass spectrometry studies of FA effects in peptides ${ }^{5,9-11}$. We conclude that the chemistry of local modifications is fundamentally different from that of long-range cross-linking. Whereas a $12 \mathrm{Da}$ reaction is the most prevalent for local modifications, a $24 \mathrm{Da}$ reaction dominates cross-linking.

In situ FA cross-linking of human cell cultures. With this clear understanding of the $24 \mathrm{Da}$ cross-linking reaction, we attempt to identify FA cross-links from in situ cross-linking experiments on intact human cells. PC9 adenocarcinoma cells were incubated in $1 \%, 2 \%, 3 \%, 4.5 \%$, or $6 \% \mathrm{FA}$ solutions for $10 \mathrm{~min}$. After the FA was washed out, the cells were lysed and the protein content prepared for mass spectrometry. We measured $10 \%$ of the peptide digest from each FA concentration directly in the mass spectrometer. The other $90 \%$ were enriched for cross-linked peptides using $\mathrm{SCX}^{27}$, and then measured in the mass spectrometer. Standard proteomics analysis identified in the digests a set of 1692 proteins with medium-to-high abundance. In order to speed up the search for cross-links, we took advantage of the complete dissociation of the FA cross-link during MS/MS fragmentation, which allows matching each peptide to the fragments independently of the other in the pair. An application implementing this strategy analyzed each mass spectrometry run against the database of the 1692 proteins in about 5 min ("Methods").

Overall, the in situ cross-linking experiments involved 59 datadependent mass spectrometry runs. The analyses of these runs searched for two separate cross-linker masses: 12 and $24 \mathrm{Da}$. We then pooled together all the identifications from these analyses into a non-redundant list of 559 cross-links (Supplementary Data 3). The false-detection rate for this list of cross-links was estimated to be $3 \%$ of the entire list, and $16 \%$ of the inter-protein list. The false-detection rate estimation was based on decoy analysis that spiked the search database with reversed sequences ("Methods"). The 24 and $12 \mathrm{Da}$ cross-linking reactions accounted for 74 and $26 \%$ of the cross-links, respectively. This reaffirms the dominance of the $24 \mathrm{Da}$ reaction in FA cross-linking also in the case of in situ FA applications. Interestingly, the $12 \mathrm{Da}$ reaction is more prevalent in situ than it was for the mixture of purified proteins, possibly reflecting influences of the cellular environment on its efficiency.

The identified cross-links occur within a subset of 276 proteins that are of relatively high abundance in the PC9 cell line ${ }^{28}$. This is expected because we did not enrich for any particular protein. Encouragingly, the cross-linked proteins originate from the nucleus (histones), cytoplasm (ribosomes and TRiC/CCT), mitochondria (HSP60), and endoplasmic reticulum (BiP), indicating that the FA has reached most cellular compartments. We could map 280 of the cross-links onto solved atomic structures. Figure $4 \mathrm{a}$ shows the histogram of the minimal $\mathrm{Ca}-\mathrm{Ca}$ distances spanned by these cross-links. The histogram includes only cross-links between two peptides that are not consecutive along the protein sequence. The FA cross-links fit the atomic structures well, having a minimal Ca-Ca distance below $25 \AA$ for $97 \%$ of them (272 cases).

Of the 559 cross-links, 90 (16\%) are inter-protein (between two different proteins in a complex) and the rest are intra-protein (within the same protein polypeptide). A subset of 28 interprotein cross-links had no corresponding atomic structures, but they showed strong indications of being true positives. All had good fragmentation of both peptides (20 fragments or more on the weakest peptide), and most were previously reported to be part of a protein complex (Table 1). These cross-links provide structural data- of in situ origin-on the relevant interactions. Particularly, each cross-link narrows down the interaction site to the vicinity of the two linked peptides.

We highlight two subsets of cross-links, which were employed for constrained docking. The first subset involves the binding site of the nascent polypeptide-associated (NAC) complex on the ribosome. Previously, Pech et al. ${ }^{29}$ showed that a conserved region in $\beta \mathrm{NAC}$, which is predicted to form an $\alpha$-helix, is binding with the ribosome. Two in situ cross-links cover this sequence region, and link it to the C-terminal of ribosomal protein $\mathrm{L} 22$. We applied PatchDock ${ }^{30}$ with the restraints of the cross-links, to dock a model of that region onto the ribosome. The best scoring model (Fig. 4b) was close to two ribosomal proteins L22 and L31, a binding mode that is consistent with previous in vitro evidence showing $\beta \mathrm{NAC}$ to also interact with $\mathrm{L} 31^{29}$.

A larger subset of cross-links mapped the interaction sites of several actin regulators onto the outer surface of the actin filament (Fig. 4c). This is consistent with their functions in regulation of bundling and bifurcation of the filaments. We performed all-atom docking onto the actin filament of plastin-2, for which a reliable homology model of the actin-binding $\mathrm{CH}$ domain could be built. This docking was restrained by the crosslink between actin and plastin-2. Remarkably, the model that ranked third by its PatchDock score had a $3.2 \AA$ deviation from a recent cryo-EM structure of filamin A (Fig. 4d), which is homologous to plastin. The available cryo-EM structures 31,32 were determined from in vitro reconstruction of actin filaments with a large excess of filamin A. Thus, our docking result provides in situ support for the relevance of the cryo-EM structure. Moreover, it suggests that the binding of filamin and plastin to the actin filament are very similar.

In contrast to the cross-links in Table 1, a subset of nine interprotein cross-links had two different indications of being false positives. First, they had marginal MS/MS fragmentation evidence (14-19 fragments on the weaker peptide in the pair). 

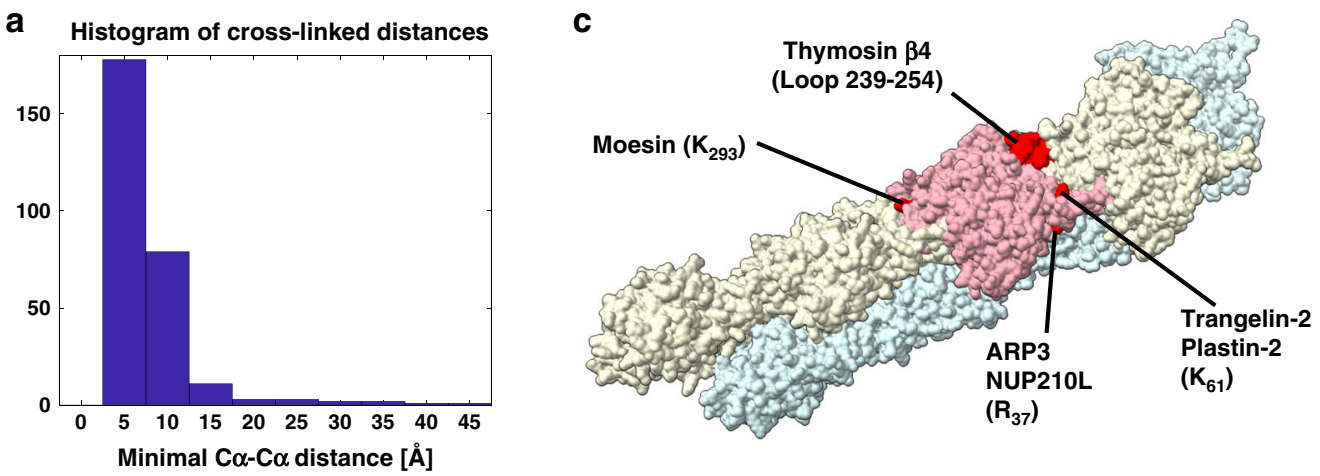

b
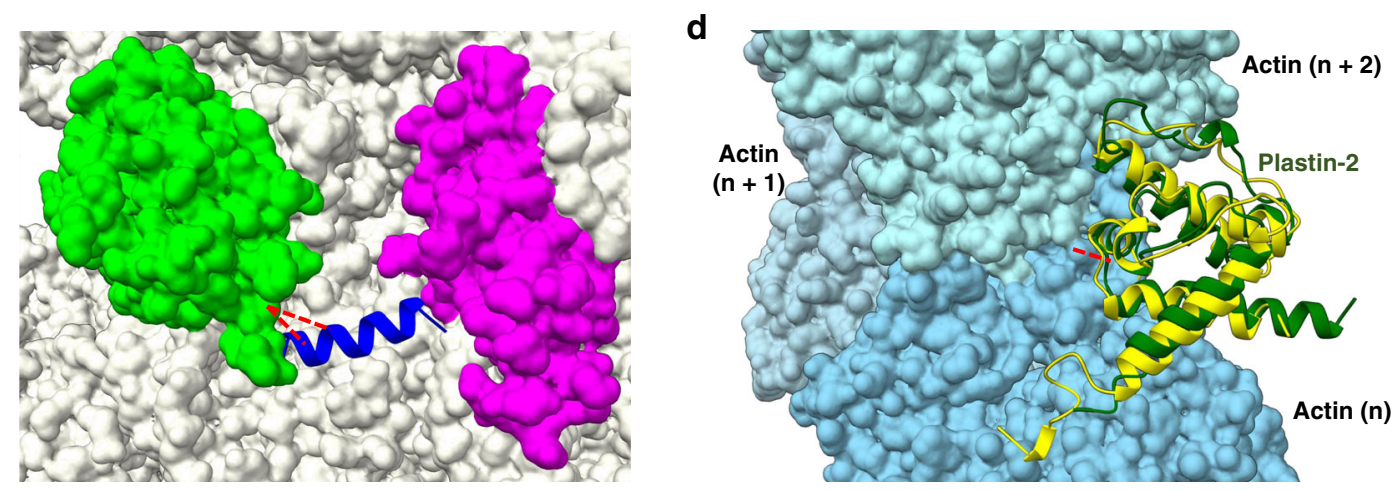

Fig. 4 In situ FA cross-linking of human PC9 cells in culture. a Histogram of the distances spanned by in situ cross-links in cases that could be mapped onto solved atomic structures. The PDB IDs used for calculating these distances are listed in Supplementary Data 3 for each cross-link. b Docking model of the binding helix of $\beta \mathrm{NAC}$ (blue) on the outer surface of the $\mathrm{S} 80$ ribosome (PDBid 6EKO ${ }^{39}$ ). The docking was constrained by two cross-links (red) to ribosomal protein L22 (green). Ribosomal protein L31 (magenta) was shown previously to interact with $\beta$ NAC as well ${ }^{29}$. c A view of the actin filament (PDBid 6D8C ${ }^{31}$ ), with one of its monomers marked in pink. Cross-links between actin and several of its regulators all map to the outer surface of the actin filament (red). $\mathbf{d}$ Docking model (green) of the $\mathrm{CH} 3$ domain of plastin-2 onto the actin filament. The docking was constrained by a single cross-link (red) between plastin-2 and actin. The model is similar ( $3.2 \AA$ RMSD) to a recent cryo-EM structure (PDBid 6 D8 $3 C^{31}$ ) of the homologous protein filamin $A$ (yellow), which was assembled on the filament in vitro. Source data are provided as a Source Data file.

Table 1 Inter-protein cross-links that provide new in situ structural information.

\begin{tabular}{|c|c|c|c|c|c|}
\hline System & Protein 1 & Protein 2 & Peptide 1 & Peptide 2 & $\begin{array}{l}\text { Reference for the } \\
\text { interaction }\end{array}$ \\
\hline \multirow{2}{*}{$\begin{array}{l}\text { Ribosome + } \\
\text { NAC }\end{array}$} & BTF3 ( $\beta$ NAC) & RL22 & $\mathrm{E}_{47} \mathrm{TIMNQEKLAK}_{57}$ & 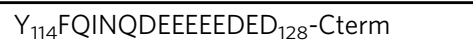 & 40 \\
\hline & BTF3 ( $\beta$ NAC) & RL22 & $\mathrm{L}_{55} \mathrm{AKLQAQVR}_{63}$ & $\mathrm{Y}_{114}$ FQINQDEEEEEDED ${ }_{128}$-Cterm & 40 \\
\hline \multirow[t]{6}{*}{ Actin } & Actin & Transgelin-2 & $D_{51}$ SYVGDEAQSKR $_{62}$ & $\mathrm{Y}_{103}$ GINTTDIFQTVDLWEGK $_{120}$ & 41 \\
\hline & Actin & Arp3 & $A_{29} V_{F P S I V G R P R} 39$ & $\mathrm{~V}_{51}$ MKGVDDLDFFIGDEAIEKPTYATK $_{75}$ & 42 \\
\hline & Actin & Thymosin $\beta-4$ & $\mathrm{~S}_{239}$ YELPDGQVITIGNER 254 & $\mathrm{E}_{33} \mathrm{TIEQEKQAGES}_{44}$-Cterm & 45 \\
\hline & Actin & NUP210L & $\mathrm{A}_{29} \mathrm{VFPSIVGRPR}_{39}$ & $\mathrm{~A}_{92} \mathrm{VLIAESTQPIR}_{103}$ & New \\
\hline & Actin-Beta & $\begin{array}{l}\text { Actin- } \\
\text { cytoskeletal }\end{array}$ & $\mathrm{I}_{192} \mathrm{LTERGYSFTTTAER}_{206}$ & $\mathrm{~V}_{98} \mathrm{APEEHPTLLTEAPLNPKANR}_{118}$ & 46 \\
\hline & $\alpha$-Actinin 4 & $\alpha$-Actinin 1 & $\mathrm{D}_{758} \mathrm{AKGISQEQMQEFR} \mathrm{R}_{771}$ & $\mathrm{~L}_{345} \mathrm{RLSNRPAFMPSEGR}_{359}$ & New \\
\hline Condensin & Smc4 & Smc2 & $\mathrm{S}_{491}$ VNEARSK $_{498}$ & $\mathrm{~N}_{407} \mathrm{DISKAQTEAK}_{417}$ & 47 \\
\hline TMED & TMED9 & TMED5 & $\mathrm{Q}_{170}$ LVEQVEQIQK $_{180}$ & $\mathrm{~L}_{153}$ EDILESINSIKSR $_{166}$ & 48 \\
\hline
\end{tabular}

Second, the two cross-linked proteins had never been reported in the literature to be interacting. Assuming that all the intra-protein cross-links are correct, then these nine cross-links are the only false positives in the entire list. As they comprise $1.6 \%$ of the list (9 out of 559), this is in accord with our a priori estimation of the false-detection rate.

\section{Discussion}

We have established four features of long-range FA cross-links in proteins. First, they occur only in structured proteins. Hence, the reliance of previous studies on peptide assays incorrectly classified the prevalent $12 \mathrm{Da}$ modification as a cross-link. Second, the dominant cross-linking reaction involves two carbon atoms 
a

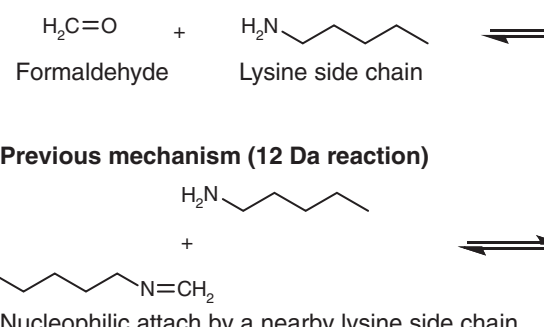

Nucleophilic attach by a nearby lysine side chain

C Proposed mechanism (24 Da reaction)

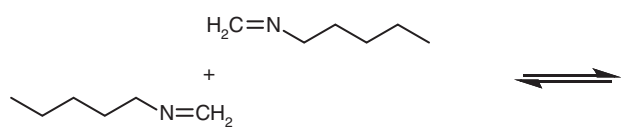

Two modified lysine side chains in structural proximity
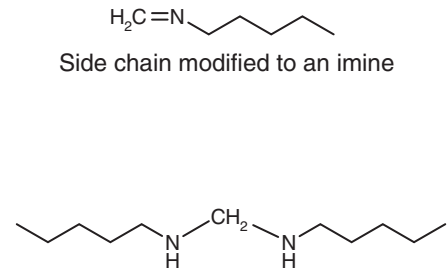

Cross-linked side chains

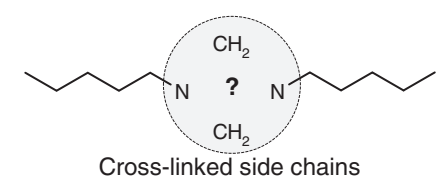

Fig. 5 Proposed mechanism for the 24 Da cross-linking reaction. The mechanism is demonstrated on two lysine side chains. a Formaldehyde modifies the primary amine of a lysine side chain into an imine. These modifications are well established by mass spectrometry. b Previously, it was thought that the cross-link is a methylene bridge that forms between a lysine side chain and a nearby imine modification. c Our data strongly suggest that formaldehyde cross-links are mainly the dimerization product of two modified side chains. The dimerization step is presumably slow and can occur only in structured proteins, in which the two side chains are close and nearly stationary relative to each other. The current data cannot conclusively determine the chemical structure at the linkage site.

(24 Da) and not one. Third, these cross-links are very labile and cleave completely under MS/MS fragmentation. Finally, the most intense MS/MS fragmentation products carry an unusual $12 \mathrm{Da}$ modification. We believe that all these factors have contributed to the fact that the chemistry of the long-range FA cross-link has not been characterized correctly.

In light of the findings, we suggest the following mechanism of FA cross-linking (Fig. 5). The reaction starts with the accepted imine formation on the side chains of lysines. The imine formation is in accord with the prevalent $12 \mathrm{Da}$ modification that others and we have observed on peptides and proteins. However, the cross-link itself forms by a dimeric interaction of two imines $^{33}$. This symmetric formation is compatible with three observations. First, it explains the symmetrical cleavage of the link under MS/MS fragmentation. Second, if one assumes that the imine modification is only mildly reactive, then it is clear why cross-linking occurs only in structured proteins: the stable structure of the protein keeps the modifications in proximity for sufficient time for cross-linking to occur. Third, the dimerization is consistent with the known reversibility of FA cross-linking, which implies that all steps of the mechanism are reversible. In particular, the MS/MS spectra clearly demonstrate the full reversal of the last dimerization step by the introduction of mild collision energy.

In Fig. 5, the cross-linking mechanism is exemplified on two lysine side chains, but FA cross-linking does not necessarily require two lysines. Indeed, for many of the in situ cross-links (Supplementary Data 3) one of the peptides has no lysine residues. Therefore, the hypothesized model would have to be revised for cross-linking in the more general case. The current data cannot conclusively determine what is the chemical structure of the linkage site. One possibility is that the two imines undergo cycloaddition to form a 1,3-diazetidine linkage. Such a strained ring structure would be consistent with the tendency of the link to break completely under HCD fragmentation. Nonetheless, other chemical structures are equally possible and efforts to better characterize the linkage site by NMR are ongoing.

In our experience, FA is not a more potent reagent compared with reagents based on NHS-esters. Yet, it has several advantages, notably its solubility and proven ability to penetrate cells and tissues rapidly. This makes FA an attractive reagent for in situ
XL-MS, which is currently not as developed as XL-MS applications on purified protein solutions or lysates. We believe that the findings of this work will now allow for a much wider use of FA for in situ XL-MS experiments.

\section{Methods}

Cross-linking of the three-protein mixture. A mixture solution of three purified proteins was prepared by reconstituting lyophilized protein powder in PBS (all reagents were purchased from Sigma unless noted otherwise). The proteins were bovine serum albumin (BSA), Ovotransferrin, and $\alpha$-Amylase with respective final molarity in the mixture of 10,10 , and $20 \mu \mathrm{M}$. Each cross-linking experiment occurred in $108 \mu \mathrm{L}$ of solution comprising a total protein mass of $260 \mu \mathrm{g}$. In most experiments we cross-linked with a formalin solution (37\% FA and $10 \%$ methanol) from Sigma (product number F8775). We also tested formalin with the same composition from another brand (DAEJUNG chemicals, Korea, product number 4044-4400). The formalin was incubated with the protein mixture at the desired FA concentration and the cross-linking reaction occurred at room temperature under gentle agitation. The cross-linking incubation time was $20 \mathrm{~min}$. The cross-linking reaction was quenched by addition of ammonium bicarbonate to a final concentration of $0.5 \mathrm{M}$ for $10 \mathrm{~min}$ before proceeding to mass spectrometry preparation. The results of each experimental condition are an average of six mass spectrometry runs from three experimental replicates, each with two technical replicates.

Cross-linking of digest from the three-protein mixture. Peptide digest was prepared from the three-protein mixture by trypsin digestion as described in the Mass spectrometry subsection ahead. The peptides were desalted on SepPak C18 column (Waters), eluted, dried in SpeedVac, and reconstituted in PBS. FA was added to a concentration of $2 \%$ and the incubation time was either $20 \mathrm{~min}, 2 \mathrm{~h}$, or $24 \mathrm{~h}$. The solution was quenched by addition of ammonium bicarbonate to a final concentration of $0.5 \mathrm{M}$ for $10 \mathrm{~min}$. The peptides were desalted on C18 stage tips and eluted for mass spectrometry analysis. The results of each incubation time are an average of two experimental replicates, each with two technical replicates.

In situ cross-linking of PC9 cells. Human lung cancer cell line PC9 (ECACC catalog No. 90071810) were seeded in Dulbecco's modified Eagle's medium, and were supplemented with $1 \times$ penicillin-streptomycin (Gibco Invitrogen) and 10\% fetal bovine serum (Biological Industries) at $37^{\circ} \mathrm{C}$ under $5 \% \mathrm{CO}_{2} / 95 \%$ air. The cells were grown to $80 \%$ confluency in $10-\mathrm{cm}$ plates. The growth media was removed and the cells washed three times with $3 \mathrm{ml}$ of warm PBS buffer. We added to each plate $2 \mathrm{ml}$ of PBS with FA at different concentrations: 1, 2, 3, 4.5, or 6\%. The cells were incubated with FA for $15 \mathrm{~min}$ at $37^{\circ} \mathrm{C}$, and then washed three times with cold PBS to remove the FA. We incubated the cells with hypertonic buffer $(50 \mathrm{mM}$ HEPES $\mathrm{pH}=7.5,500 \mathrm{mM} \mathrm{NaCl}, 0.5 \mathrm{mM}$ EDTA, $0.0005 \%$ Tween20) for $15 \mathrm{~min}$, and then scraped the cells from the plate. The cells were centrifuged at $4{ }^{\circ} \mathrm{C}$ and the supernatant was discarded. The cell pellet was resuspended for $15 \mathrm{~min}$ with hypotonic buffer (above buffer without $\mathrm{NaCl}$ ), and then further lysed with sonication ( $5 \mathrm{~s}$ on, $25 \mathrm{~s}$ off, 5 times, $50 \%$ amplitude). The cell lysate was centrifuged at 
$4^{\circ} \mathrm{C}$ and the supernatant was collected. The lysate was processed by the filter-aided sample preparation protocol ${ }^{34}$ in order to remove the detergent and nucleic acids prior to the mass spectrometry analysis.

Enrichment by strong cation exchange (SCX) chromatography. We followed the SCX protocol by Klykov et al. ${ }^{27}$. Briefly, desalted peptide digest was dried in SpeedVac and reconstituted in $50 \mu \mathrm{l}$ of buffer A (20\% Acetonitrile, formic acid titrated to $\mathrm{pH}$ of 3.0). Separation was performed with an Äkta Pure system on a $100 \times 1.0 \mathrm{~mm}$ PolySULFOETHYL A SCX column (PolyLC, USA) using a gradient of buffer B (20\% Acetonitrile, $0.5 \mathrm{M} \mathrm{NaCl}$, formic acid titrated to $\mathrm{pH}$ of 3.0$)$ and $100 \mu \mathrm{l}$ fractions. Fractions corresponding to $\mathrm{NaCl}$ concentrations of $100 \mathrm{mM}$ and higher were desalted and used for mass spectrometry analysis.

Mass spectrometry. The proteins were precipitated in acetone at $-80^{\circ} \mathrm{C}$ for $1 \mathrm{~h}$ followed by centrifugation at $10,000 \times \mathrm{g}$. The pellet was resuspended in $20 \mu \mathrm{l}$ of $8 \mathrm{M}$ urea with $10 \mathrm{mM}$ DTT. After $30 \mathrm{~min}$, iodoacetamide was added to a final concentration of $50 \mathrm{mM}$ and the alkylation reaction proceeded for $30 \mathrm{~min}$. The urea was diluted by adding $200 \mu \mathrm{l}$ of digestion buffer ( $25 \mathrm{mM}$ TRIS $\mathrm{pH}=8.0 ; 10 \%$ acetonitrile), trypsin (Promega) was added at a 1:100 protease-to-protein ratio, and the protein was digested overnight at $37^{\circ} \mathrm{C}$ under agitation. Following digestion, the peptides were desalted on C18 stage tips and eluted by 55\% acetonitrile. The eluted peptides were dried in a SpeedVac, reconstituted in $0.1 \%$ formic acid, and measured in the mass spectrometer. The samples were analyzed by a $120 \mathrm{~min}$ $0-40 \%$ acetonitrile gradient on a liquid chromatography system coupled to a QExactive Plus mass spectrometer (Thermo). We were careful not to raise the temperature of the sample above $40^{\circ} \mathrm{C}$ through all the preparation stages (alkylation, digestion, desalting, and in the analytical column of the LC) in order not to break the FA cross-links. The RAW data files from the mass spectrometer were converted to MGF format by Proteome Discoverer (Thermo), which was the input format for our analysis applications. The method parameters of the run were as follows: data-dependent acquisition; Full MS resolution 70,000; MS1 AGC target le6; MS1 Maximum IT $200 \mathrm{~ms}$; Scan range 450-1800; dd-MS/MS resolution 35,000; MS/MS AGC target 2e5; MS2 Maximum IT 300 ms; Loop count Top 12; Isolation window 1.1; Fixed first mass 130; MS2 Minimum AGC target 800; HCD energy (NCE) 26;Charge exclusion: unassigned,1,2,3,8,>8; Peptide match-off; Exclude isotope-on; Dynamic exclusion $45 \mathrm{~s}$.

Scanning for the mass of the cross-linking reaction. We modified our analysis application, FindXL ${ }^{35}$, so that it ran multiple times, each time with a different cross-linker mass. We scanned all the integer masses from -30 to $50 \mathrm{Da}$. FindXL exhaustively enumerates all the possible peptide pairs and compare them to the measured MS/MS events in search of matches that fulfill the criteria below. The search parameters were as follows: Sequence database-the sequences of BSA, Ovotransferrin, and $\alpha$-Amylase; Protease-trypsin, allowing up to three miscleavage sites; Fixed modification of cysteine by iodoacetamide; Variable modification of methionine by oxidation; Cross-linking can occur on any residue type; Cross-linker is non-cleavable; MS/MS fragments to consider-b-ions and y-ions as well as b-ions and y-ions with the additional mass of the second peptide and the cross-linker; $\mathrm{MS}^{1}$ tolerance $-6 \mathrm{ppm}$; $\mathrm{MS}^{2}$ tolerance- $8 \mathrm{ppm}$.

A cross-link was identified as a match between a MS/MS event and a peptide pair if it fulfilled four conditions: (1) The mass of the precursor ion is the same as the expected mass of the cross-linked peptide pair within the $\mathrm{MS}^{1}$ tolerance; (2) At least four MS/MS fragments (within the $\mathrm{MS}^{2}$ tolerance) were identified on each peptide; (3) The fragmentation score of the cross-link (defined as the number of matching MS/MS fragments divided by the combined length of the two peptides) is 0.6 or higher; (4) The peptides are not overlapping nor consecutive in the protein sequence. The purpose of the fourth criterion is to count only cross-links that span a long range on the primary structure.

Identifying the amino acids involved in the $24 \mathrm{Da}$ reaction. The identified crosslinks from all the replicates involving 2 and $4 \%$ FA cross-linking were pooled together for this analysis. For each cross-link, we analyzed the two peptides independently of each other. For each peptide, we computationally modified (added $12 \mathrm{Da}$ ) in turn to each residue. We then determined which residue position was most compatible with the MS/MS fragmentation pattern (highest number of fragments that can be assigned by the modified peptide at $8 \mathrm{ppm}$ tolerance). The number of times each amino acid was found to be the most compatible was then normalized by dividing it by the total number of occurrences of that amino acid in all the peptides (normalized count).

Identifying linear peptides with modifications. The identification of modifications formed by FA on linear peptides was based only on matching the mass of the precursor ion (i.e., $\mathrm{MS}^{1}$ ) to the theoretical mass of the peptide+modification. This approach was taken because of insufficient knowledge as to where these modifications occur, or how they affect the MS/MS fragmentation. To make the identification more stringent, we set a very narrow tolerance of $1 \mathrm{ppm}$ on the match between the measured and theoretical mass of the peptide plus the modification. Of note, with such a narrow tolerance we did not find any ambiguous cases in which the measured mass could be assigned to more than one peptide. We ran the analysis eight times, each time searching for a different modification: 0.0 (no modification), $12.0,24.0,36.0,48.0,60.0,57.0215$ (off-target alkylation), and 15.9949 (oxidation) $\mathrm{Da}$. The estimate of the relative abundance of each modification was calculated as the ratio between the number of identified peptides with that modification and the number of identified peptides without modification $(0.0 \mathrm{Da})$. Other search parameters were: Sequence database-the sequences of BSA, Ovotransferrin, and aAmylase; Protease-trypsin, allowing up to three miscleavage sites; Fixed modification of cysteine by iodoacetamide. Methionine oxidation was not considered.

Cross-link identification in a small set of proteins. This analysis application exhaustively enumerates all the possible peptide pairs, and compare them to the measured MS/MS events in search of matches that fulfill the criteria below. The search parameters were as follows: Sequence database-the sequences of BSA, Ovotransferrin, and $\alpha$-Amylase; Protease-trypsin, allowing up to three miscleavage sites; Fixed modification of cysteine by iodoacetamide; Variable modification of methionine by oxidation; Cross-linking can occur on any residue type; Cross-linker is always cleaved; MS/MS fragments to consider: $\mathrm{b}$-ions, $\mathrm{y}$-ions, ${ }^{*} \mathrm{~b}$-ions (b-ions plus 12.0 Da), and ${ }^{*} \mathrm{y}$-ions (y-ions plus $12.0 \mathrm{Da}$ ); $\mathrm{MS}^{1}$ tolerance $-6 \mathrm{ppm}$; $\mathrm{MS}^{2}$ tolerance -8 ppm; Cross-linker mass-one of three possible masses: 24.0, 25.00335, and 26.0067. The three cross-linker masses were considered in turn in the calculation of the theoretical mass of the two cross-linked peptides. These masses address the incorrect reporting of the mono-isotopic mass (Supplementary Fig. 1).

A cross-link was identified as a match between a measured MS/MS event and a peptide pair if it fulfilled five conditions: (1) The mass of the precursor ion is within the $\mathrm{MS}^{1}$ tolerance of the theoretical mass of the linked peptide pair (with either of the three possible cross-link masses); (2) At least four modified MS/MS fragments $(* \mathrm{~b}$ and $* \mathrm{y})$ were identified within the $\mathrm{MS}^{2}$ tolerance on each peptide; (3) The fragmentation score of the cross-link (defined as the number of all matching MS/ MS fragments divided by the combined length of the two peptides) is 1.0 or higher; (4) The peptides are not overlapping in the protein sequence; (5) There is no other peptide pair or linear peptide that match the data with equal or better fragmentation score.

Given the small size of the sequence database, we estimated the false-detection rate in the following way. The analysis of data from the $4 \% \mathrm{FA}$ experiment was repeated ten times with an erroneous cross-linker mass of $61.0,62.0,63.0, \ldots 70.0$ Da. This led to fragmentation scores that were much lower than the scores obtained with the correct cross-linker mass. On average, 2 erroneous cross-links had a fragmentation score above 1.0 in each decoy run, whereas runs with the correct cross-linker mass $(24.0 \mathrm{Da})$ identified $\sim 60$ cross-links above the 1.0 score We therefore estimate the false-detection rate to be 2 in 60 cross-links or $\sim 3 \%$.

Cross-link identification in a large set of proteins. This application relied on the complete cleavage of the FA cross-links in order to separately assign a MS/MS fragmentation score to each peptide. This division allows for a practical run time of $O(n)$ with suitable preprocessing. The search parameters were as follows: Sequence database-comprising the 1692 human proteins that were identified in the samples. Note that runs on the full human proteome (20,000 proteins) are possible, but take up to $4 \mathrm{~h}$; Protease-trypsin, allowing up to two miscleavage sites; Fixed modification of cysteine by iodoacetamide; Cross-linking can occur on any residue type; Cross-linker is always cleaved; MS/MS fragments to consider: b-ions, y-ions, ${ }^{*} \mathrm{~b}-$ ions (b-ions plus $12.0 \mathrm{Da}$ ), and ${ }^{*} \mathrm{y}$-ions ( $\mathrm{y}$-ions plus $12.0 \mathrm{Da}$ ); $\mathrm{MS}^{1}$ tolerance -4.2 ppm; $\mathrm{MS}^{2}$ tolerance $-6.5 \mathrm{ppm}$; Cross-linker mass-one of five possible masses: $24.0,25.00335,26.0067,12.0$, and $13.00335 \mathrm{Da}$. All of these masses were considered in turn in the calculation of the theoretical mass of the two cross-linked peptides. The five masses address the incorrect reporting of the mono-isotopic mass (Supplementary Fig. 1), as well as the much less frequent $12 \mathrm{Da}$ reaction.

A cross-link was reported if it fulfilled four conditions: (1) The mass of the precursor ion is within the $\mathrm{MS}^{1}$ tolerance of the theoretical mass of the cross-linked peptide pair (with any of the five cross-link masses); (2) Each peptide had at least 19 MS/MS fragments (b, y, *b and *y) within the MS ${ }^{2}$ tolerance, OR its fragmentation score (defined as the number of matching MS/MS fragments divided by its length) was 1.8 or higher; (3) The peptides are not overlapping in the protein sequence; (4) There is no other peptide pair or linear peptide that match the data with equal or better fragmentation score.

To estimate the false-detection rate of the reported list of cross-links, we spiked the sequence database with a decoy set comprising some of the sequences in reverse. The proteins used for the decoys were chosen randomly and their number is user defined. In the case of the PC9 lysate, the number of decoy sequences was set to $1 / 15$ the total number of sequences. We therefore estimate the number of false positives in the cross-link list to be 15 times the number of cross-links that include a reverse decoy peptide.

Computational docking. Docking was performed with PatchDock ${ }^{30}$. The crosslink was implemented as distance constraints that must be under $12 \AA$ in accepted models. Homology models of $\beta$ NAC and Plastin-2 were generated by HHPred ${ }^{36}$.

Reporting summary. Further information on research design is available in the Nature Research Reporting Summary linked to this article. 


\section{Data availability}

The mass spectrometry data have been deposited to the ProteomeXchange Consortium via the PRIDE $^{37}$ partner repository with the dataset identifier PXD015435. Source data are provided with this paper. All other data are available from the corresponding author on reasonable request.

\section{Code availability}

A standalone analysis application for identification of formaldehyde cross-links is available at https://github.com/Kalisman-Lab/Search_Formaldehyde_Cross-links. The underlying source code in Java is available at https://github.com/Kalisman-Lab/ Search_Formaldehyde_Cross-links_Source_Code.

Received: 10 March 2020; Accepted: 2 June 2020;

Published online: 19 June 2020

\section{References}

1. Karnovsky, M. J. A formaldehyde-glutaraldehyde fixative of high osmolality for use in electron microscopy. J. Cell Biol. 27, 137-138A (1965).

2. Carson, F. L., Martin, J. H. \& Lynn, A. J. Formalin fixation for electron microscopy: a re-evaluation. J. Clin. Pathol. 59, 365-373 (1973).

3. Solomon, M. J. \& Varshavsky, A. Formaldehyde-mediated DNA-protein crosslinking: a probe for in vivo chromatin structures.. PNAS 82, 6470-6474 (1985).

4. Chang, Y. T. \& Loew, G. H. Reaction mechanisms of formaldehyde with endocyclic imino groups of nucleic acid bases. J. Am. Chem. Soc. 116, 3548-3555 (1994).

5. Metz, B. et al. Identification of formaldehyde-induced modifications in proteins reactions with model peptides. J. Biol. Chem. 279, 6235-6243 (2004).

6. Hoffman, E. A., Frey, B. L., Smith, L. M. \& Auble, D. T. Formaldehyde crosslinking: a tool for the study of chromatin complexes. J. Biol. Chem. 290, 26404-26411 (2015).

7. Fraenkel-Conrat, H. \& Olcott, H. S. The reaction of formaldehyde with proteins. V. Cross-linking between amino and primary amide or guanidy groups. J. Am. Chem. Soc. 70, 2673-2684 (1948).

8. Feldman, M. Y. Reactions of nucleic acids and nucleoproteins with formaldehyde. Prog. Nucleic Acid Res. Mol. Biol. 13, 1-49 (1973).

9. Metz, B. et al. Identification of formaldehyde-induced modifications in proteins: reactions with insulin. Bioconjugate Chem. 17, 815-822 (2006).

10. Toews, J., Rogalski, J. C., Clark, T. J. \& Kast, J. Mass spectrometric identification of formaldehyde-induced peptide modifications under in vivo protein cross-linking conditions. Anal. Chim. Acta 618, 168-183 (2008).

11. Wang, Z. J. et al. Chemical modifications of peptides and proteins with low concentration formaldehyde studied by mass spectrometry. Chin. J. Anal. Chem. 44, 1193-1199 (2016).

12. Leitner, A., Faini, M., Stengel, F. \& Aebersold, R. Crosslinking and Mass Spectrometry: An Integrated Technology to Understand the Structure and Function of Molecular Machines. Trends Biochem Sci. 41, 20-32 (2016).

13. Schneider, M., Belsom, A. \& Rappsilber, J. Protein tertiary structure by crosslinking/mass spectrometry. Trends Biochem Sci. 43, 157-169 (2018).

14. Sinz, A. Cross-linking/mass spectrometry for studying protein structures and protein-protein interactions: where are we now and where should we go from here? Angew. Chem. Int Ed. Engl. 57, 6390-6396 (2018).

15. Herzog, F. et al. Structural probing of a protein phosphatase $2 \mathrm{~A}$ network by chemical cross-linking and mass spectrometry. Science 337, 1348-1352 (2012).

16. Rappsilber, J. The beginning of a beautiful friendship: cross-linking/mass spectrometry and modelling of proteins and multi-protein complexes. $J$ Struct. Biol. 173, 530-540 (2011)

17. Weisbrod, C. R. et al. In vivo protein interaction network identified with a novel real-time cross-linked peptide identification strategy. J. Proteome Res. 12, 1569-1579 (2013).

18. Kaake, R. M. et al. A new in vivo cross-linking mass spectrometry platform to define protein-protein interactions in living cells. Mol. Cell Proteom. 13, 3533-3543 (2014)

19. Chavez, J. D. et al. Chemical crosslinking mass spectrometry analysis of protein conformations and supercomplexes in heart tissue. Cell Syst. 6 , 136-141 (2018).

20. Fasci, D., van Ingen, H., Scheltema, R. A. \& Heck, A. J. R. Histone interaction landscapes visualized by crosslinking mass spectrometry in intact cell nuclei. Mol. Cell Proteom. 17, 2018-2033 (2018).

21. Robinson, P. J. et al. Structure of a complete mediator-RNA polymerase II preinitiation complex. Cell 166, 1411-1422 (2016).

22. Wang, X. et al. The proteasome-interacting Ecm 29 protein disassembles the $26 \mathrm{~s}$ proteasome in response to oxidative stress. J. Biol. Chem. 292, 16310-16320 (2017).
23. Lenz, S., Giese, S. H., Fischer, L. \& Rappsilber, J. In-search assignment of monoisotopic peaks improves the identification of cross-linked peptides. J. Proteome Res. 17, 3923-3931 (2018).

24. Götze, M., Iacobucci, C., Ihling, C. H. \& Sinz, A. A simple cross-linking/mass spectrometry workflow for studying system-wide protein interactions. Anal. Chem. 91, 10236-10244 (2019).

25. Sinz, A. Divide and conquer: cleavable cross-linkers to study protein conformation and protein-protein interactions. Anal. Bioanal. Chem. 409, 33-44 (2017).

26. Iacobucci, C. et al. A cross-linking/mass spectrometry workflow based on MScleavable cross-linkers and the MeroX software for studying protein structures and protein-protein interactions. Nat. Protoc. 13, 2864-2889 (2018).

27. Klykov, O. et al. Efficient and robust proteome-wide approaches for crosslinking mass spectrometry. Nat. Protoc. 13, 2964-2990 (2018).

28. Geiger, T., Wehner, A., Schaab, C., Cox, J. \& Mann, M. Comparative proteomic analysis of eleven common cell lines reveals ubiquitous but varying expression of most proteins. Mol. Cell Proteom. 11, M111.014050 (2012).

29. Pech, M., Spreter, T., Beckmann, R. \& Beatrix, B. Dual binding mode of the nascent polypeptide-associated complex reveals a novel universal adapter site on the ribosome. J. Biol. Chem. 285, 19679-19687 (2010).

30. Schneidman-Duhovny, D., Inbar, Y., Nussinov, R. \& Wolfson, H. J. PatchDock and SymmDock: servers for rigid and symmetric docking. Nucl. Acids Res. 33, W363-W367 (2005).

31. Galkin, V. E., Orlova, A., Cherepanova, O., Lebart, M. C. \& Egelman, E. H. High-resolution cryo-EM structure of the F-actin-fimbrin/plastin ABD2 complex. Proc. Natl Acad. Sci. USA 105, 1494-1498 (2008).

32. Iwamoto, D. V. et al. Structural basis of the filamin A actin-binding domain interaction with F-actin. Nat. Struct. Mol. Biol. 25, 918-927 (2019).

33. Layer, R. W. The chemistry of imines. Chem. Rev. 63, 489-510 (1965).

34. Wiśniewski, J. R., Zougman, A., Nagaraj, N. \& Mann, M. Universal sample preparation method for proteome analysis. Nat. Methods 6, 359-362 (2009).

35. Kalisman, N., Adams, C. M. \& Levitt, M. Subunit order of eukaryotic TRiC/ CCT chaperonin by cross-linking, mass spectrometry, and combinatorial homology modeling. Proc. Natl Acad. Sci. USA 109, 2884-2889 (2012).

36. Söding, J., Biegert, A. \& Lupas, A. N. The HHpred interactive server for protein homology detection and structure prediction. Nucleic Acids Res. 33, W244-W248 (2005).

37. Perez-Riverol, Y. et al. The PRIDE database and related tools and resources in 2019: improving support for quantification data. Nucleic Acids Res. 47, D442-D450 (2019).

38. Kurokawa, H. L., Mikami, B. \& Hirose, M. Crystal structure of diferric hen ovotransferrin at $2.4 \AA$ resolution. J. Mol. Biol. 254, 196-207 (1995).

39. Natchiar, S. K., Myasnikov, A. G., Kratzat, H., Hazemann, I. \& Klaholz, B. P. Visualization of chemical modifications in the human $80 \mathrm{~s}$ ribosome structure. Nature 551, 472-477 (2017)

40. Beatrix, B., Sakai, H. \& Wiedmann, M. The $\alpha$ and $\beta$ subunit of the nascent polypeptide-associated complex have distinct functions. J. Biol. Chem. 275, 37838-37845 (2000).

41. Kobayashi, R., Kubota, T. \& Hidaka, H. Purification, characterization, and partial sequence analysis of a new $25-\mathrm{kDa}$ actin binding protein from bovine aorta: a SM22 homolog. Biochem. Biophys. Res. Commun. 198, 1275-1280 (1994).

42. Welch, M. D., Iwamatsu, A. \& Mitchison, T. J. Actin polymerization is induced by Arp2/3 protein complex at the surface of Listeria monocytogenes. Nature 385, 265-269 (1997)

43. Janji, B. et al. Phosphorylation on Ser5 increases the F-actin-binding activity of L-plastin and promotes its targeting to sites of actin assembly in cells. J. Cell Sci. 119, 1947-1960 (2006).

44. Huang, L., Wong, T. Y., Lin, R. C. \& Furthmayr, H. Replacement of threonine 558 , a critical site of phosphorylation of moesin in vivo, with aspartate activates F-actin binding of moesin. Regulation by conformational change. $J$. Biol. Chem. 274, 12803-12810 (1999).

45. Safer, D., Elzinga, M. \& Nachmias, V. T. Thymosin beta 4 and Fx, an actinsequestering peptide, are indistinguishable. J. Biol. Chem. 266, 4029-4032 (1991).

46. Hein, M. Y. et al. A human interactome in three quantitative dimensions organized by stoichiometries and abundances. Cell 163, 712-723 (2015).

47. Soh, Y. M. et al. Molecular basis for SMC rod formation and its dissolution upon DNA binding. Mol. Cell 57, 290-303 (2015).

48. Koegler, E. et al. p28, a novel ERGIC/cis Golgi protein, required for Golgi ribbon formation. Traffic 11, 70-89 (2010).

\section{Acknowledgements}

This work was supported by the Israel Science Foundation grant number 1768/15. M.S. was supported by the FSHD Global Foundation grant number 41. We thank Uri Raviv for his help and advice in various stages of this work. We thank David Morgenstern and Dina Schneidman for critical reading of the manuscript. 


\section{Author contributions}

Conceptualization, N.K.; Methodology, T.T-W., M.S., and N.K.; Investigation, T.T-W., M.S., and J.Z.; Software, A.B., S.C., and N.K.; Resources, A.M., X.S., D.E.S., and O.R.; Writing-original draft, T.T-W., and N.K.; Writing-review \& editing, D.E.S., O.R., and N.K.; Visualization, T.T-W., M.S., D.E.S., and N.K.; Supervision, O.R. and N.K.; Funding acquisition, N.K.

\section{Competing interests}

The authors declare no competing interests.

\section{Additional information}

Supplementary information is available for this paper at https://doi.org/10.1038/s41467 020-16935-w.

Correspondence and requests for materials should be addressed to N.K.

Peer review information Nature Communications thanks Michael Trnka, and the other, anonymous, reviewer(s) for their contribution to the peer review of this work. Peer reviewer reports are available.
Reprints and permission information is available at http://www.nature.com/reprint

Publisher's note Springer Nature remains neutral with regard to jurisdictional claims in published maps and institutional affiliations.

(c) Open Access This article is licensed under a Creative Commons Attribution 4.0 International License, which permits use, sharing, adaptation, distribution and reproduction in any medium or format, as long as you give appropriate credit to the original author(s) and the source, provide a link to the Creative Commons license, and indicate if changes were made. The images or other third party material in this article are included in the article's Creative Commons license, unless indicated otherwise in a credit line to the material. If material is not included in the article's Creative Commons license and your intended use is not permitted by statutory regulation or exceeds the permitted use, you will need to obtain permission directly from the copyright holder. To view a copy of this license, visit http://creativecommons.org/ licenses/by/4.0/.

(c) The Author(s) 2020 